\title{
Calcified shrimp waste supported Pd NPs as an efficient catalyst for benzene destruction
}

Tareque Odoom-Wubah, ${ }^{\mathbf{a}}$ Qun Li, ${ }^{\text {a }}$ Rafal Mulka, ${ }^{\mathbf{a}}$ Min Chen, ${ }^{\mathbf{a}}$ Jiale Huang, ${ }^{\mathbf{a} *}$ Qingbiao $i^{\mathbf{a}, \mathbf{b}}$ and Rafael Luque $\mathbf{c}^{\mathbf{d} \boldsymbol{d} *}$

aDepartment of Chemical and Biochemical Engineering, College of Chemistry and

Chemical Engineering, Xiamen University, Xiamen 361005, PR China,

b College of Food and Biological Engineering, Jimei University, Xiamen 361021, PR

China

c Department de Quimica Organica, Universidad de Cordoba, Campus de Rabanales,

Edificio Marie Curie (C-3), Ctra Nnal IV-A, Km. 396 E-14014, Cordoba (Spain).

d People's Friendship University of Russia (RUDN, University) ,6 Miklukho-Maklaya

str.,117198, Moscow, Russia.

Corresponding authors E-mail addresses:

cola@xmu.edu.cn

rafael.luque@uco.es

*Corresponding author. Tel.: (+86) 592-2183088; fax: $(+86) 592-2184822$.

E-mail addresses: cola@xmu.edu.cn (J. H.),

${ }^{*}$ Corresponding author. Tel.: (+34) 957211050; fax: $(+34) 957212066$

E-mail addresses: rafael.luque@uco.es (R.L) 


\section{Number of pages: 15}

Number of figures: 10

\section{Number of tables: 5}

\section{Table of contents}

Figure S 1. BET surface area showing (a) pore diameter (b) and (c) of the Pd/SW catalysts................... 3

Figure S 2. TEM and corresponding SEM images (a) Pd/SW@400 (b)Pd/SW@600 (c) Pd/SW@800 and (d) $\mathrm{Pd} / \mathrm{SW} @ 900$

Figure S 3. XPS spectra of the Pd/SW catalysts at different Pd wt $\%$ loading (a) Pd $3 \mathrm{~d}$ (b) Ca 2 p region........S 5

Figure S 4. XPS spectra C $1 \mathrm{~s}$ region (a-d) and N $1 \mathrm{~s}$ region (e-h) of the $\mathrm{Pd} / \mathrm{SW}$ catalyst.................... 6

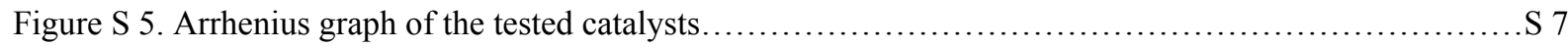

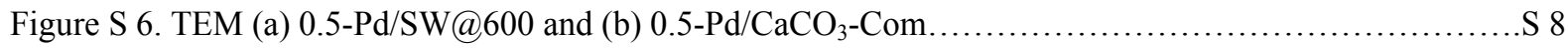

Figure $\mathrm{S}$ 7. Comparison of the $\mathrm{H}_{2}$-TPR (a and c) $\mathrm{O}_{2}$-TPD ( $b$ and d) and $\mathrm{C}_{6} \mathrm{H}_{6}-\mathrm{TPD}$ of the 0.5-Pd/SW@600 and 0.5-



Figure S 8. XRD (a) and XPS analysis showing the (b) Pd 3d (c) Ca 2p and O1s region of the 0.5-Pd/SW@600



Figure S 9. TEM (a) and SEM analysis of the $0.5-\mathrm{Pd} / \mathrm{SW} @ 600$ catalyst after $120 \mathrm{~h}$ of reaction at $250^{\circ} \mathrm{C}$ and $\mathrm{GHSV}=$



Figure S 10. Effect of $\mathrm{CO}_{2}$ on conversion (a) and reuse-ability tests (b) of the 0.5-Pd/SW@600 catalyst ( $0.05 \mathrm{~g}$ of catalyst, $1000 \mathrm{ppm}$ of benzene, $20 \% \mathrm{O}_{2}$ and balance $\mathrm{N}_{2}, \mathrm{CO}_{2}$ on conversion was done at $\mathrm{GHSV}=60,000$ $\mathrm{mL} \cdot \mathrm{g}^{-1} \cdot \mathrm{h}^{-1}$ and the re-usability test GHSV $=20,000 \mathrm{~mL} \cdot \mathrm{g}^{-1} \cdot \mathrm{h}^{-1}$ ) N.B Figure S10a was conducted in the presence of 10 and $20 \mathrm{vol} \%$ of high purity $\mathrm{CO}_{2}$. Before each reuse-ability test the temperature was allowed to cool down to $100{ }^{\circ} \mathrm{C}$. S 11

Table S 1. Binding energies of the $\mathrm{Pd} 3 \mathrm{~d}, \mathrm{Ca} 2 \mathrm{p}$ and $\mathrm{O} 1 \mathrm{~s}$ region of different $\mathrm{Pd} / \mathrm{SW}$ catalysts..................S12





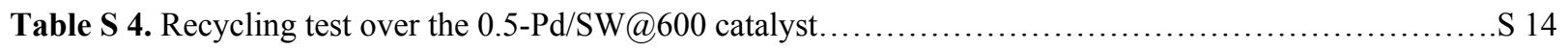

Table S 5. Comparison of the 0.5-Pd/SW@600 with others in literature.........................................S14 

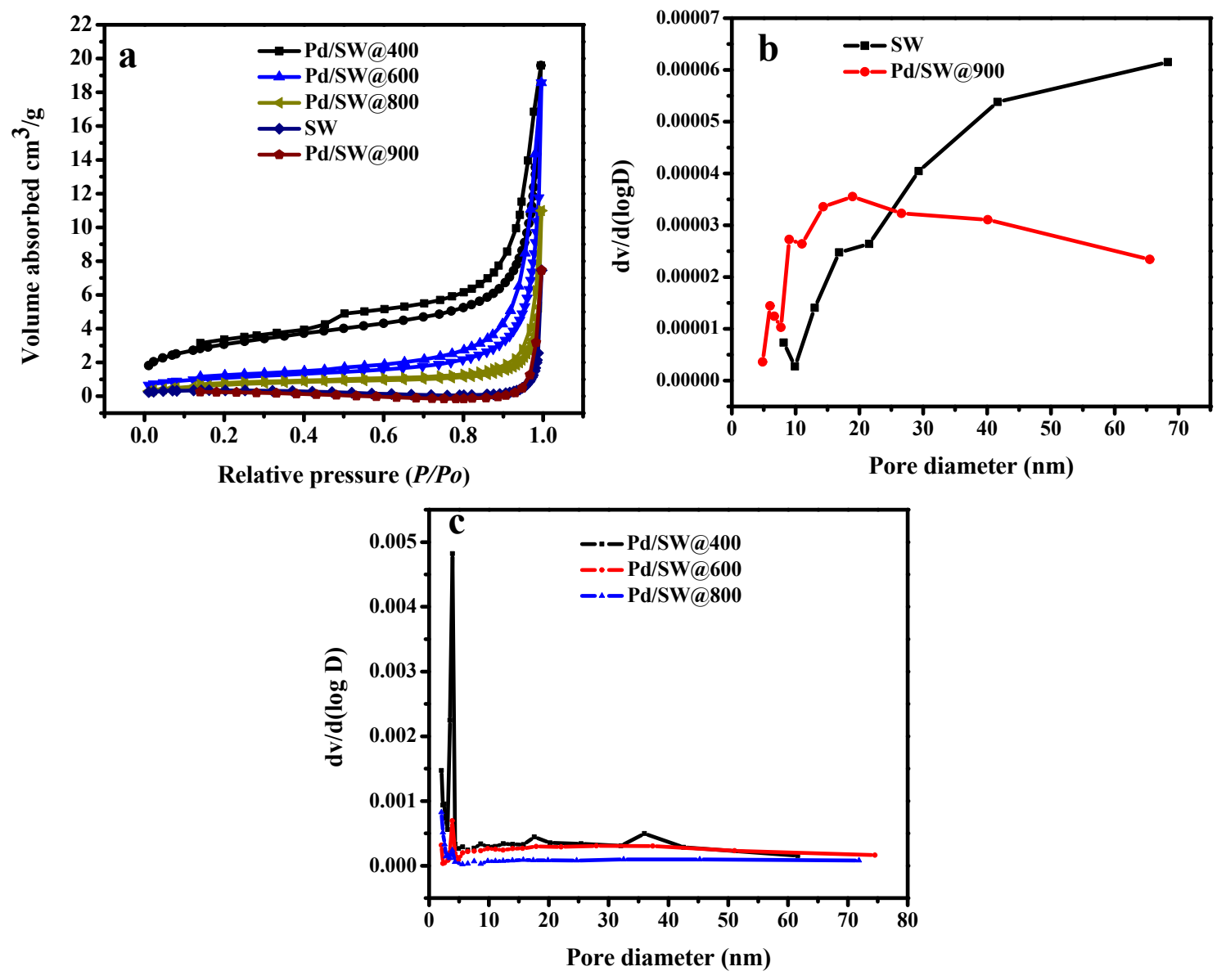

Figure S 1. BET surface area showing (a) pore diameter(b) and (c) of the $\mathrm{Pd} / \mathrm{SW}$ catalysts 

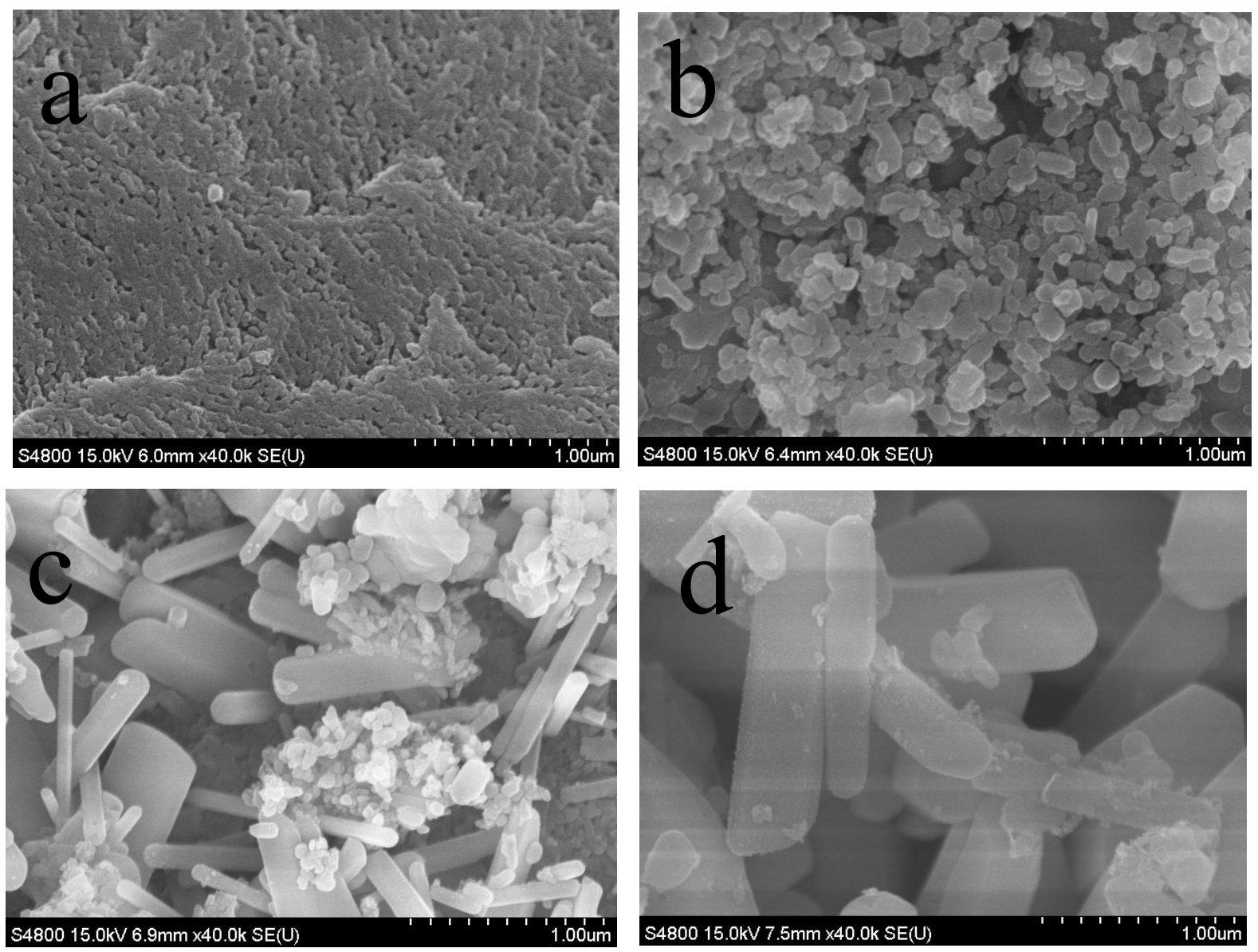

Figure S 2. TEM and corresponding SEM images (a) Pd/SW@400 (b)Pd/SW@600 (c) Pd/SW@800 and (d) Pd/SW@900 



Figure S 3.XPS spectra of the $\mathrm{Pd} / \mathrm{SW}$ catalysts at different $\mathrm{Pd} w \mathrm{w} \%$ loading (a) $\mathrm{Pd} 3 \mathrm{~d}$ (b) $\mathrm{Ca} 2 \mathrm{p}$ region 

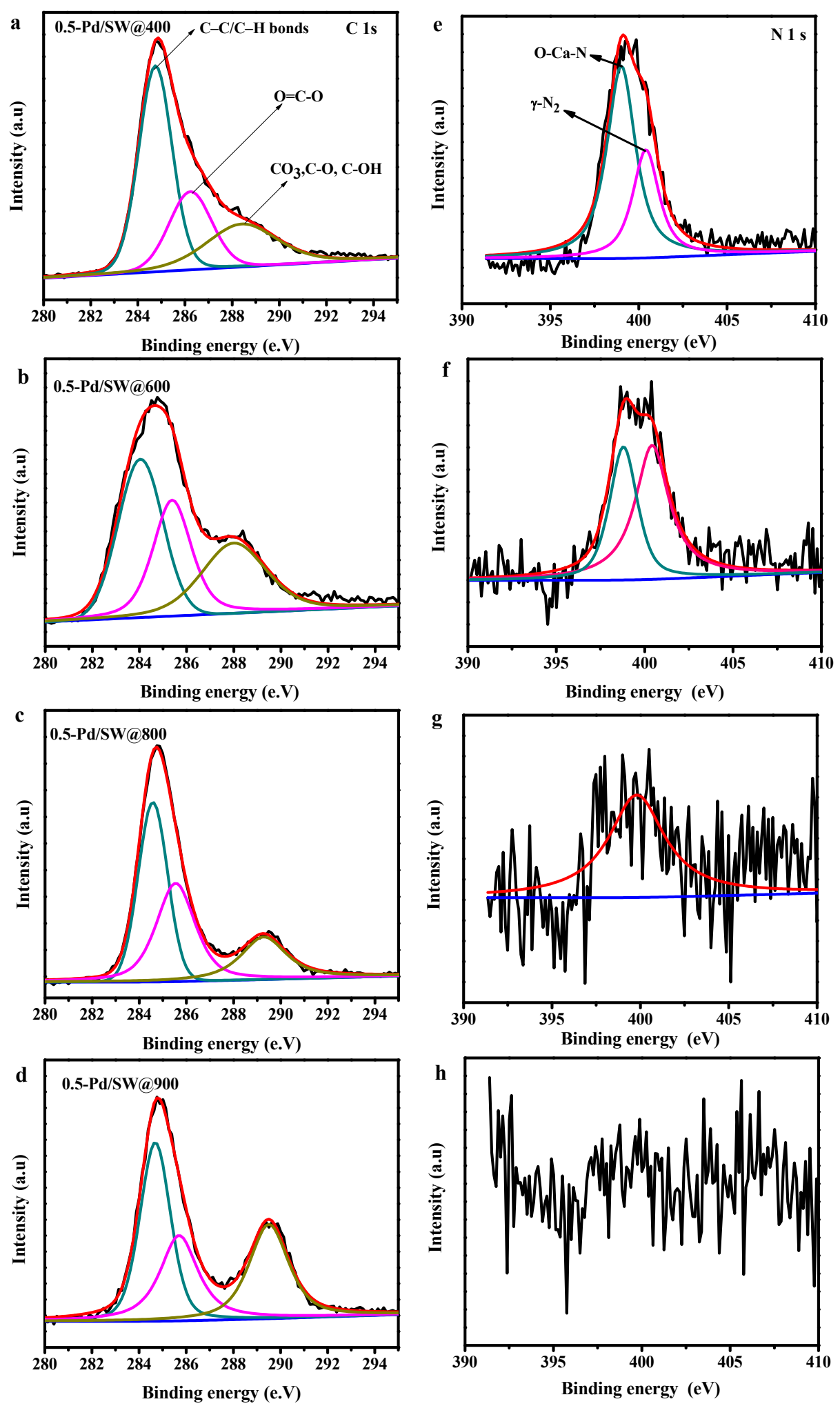

Figure S 4. XPS spectra C 1 s region (a-d) and N 1 s region (e-h) of the Pd/SW catalyst 

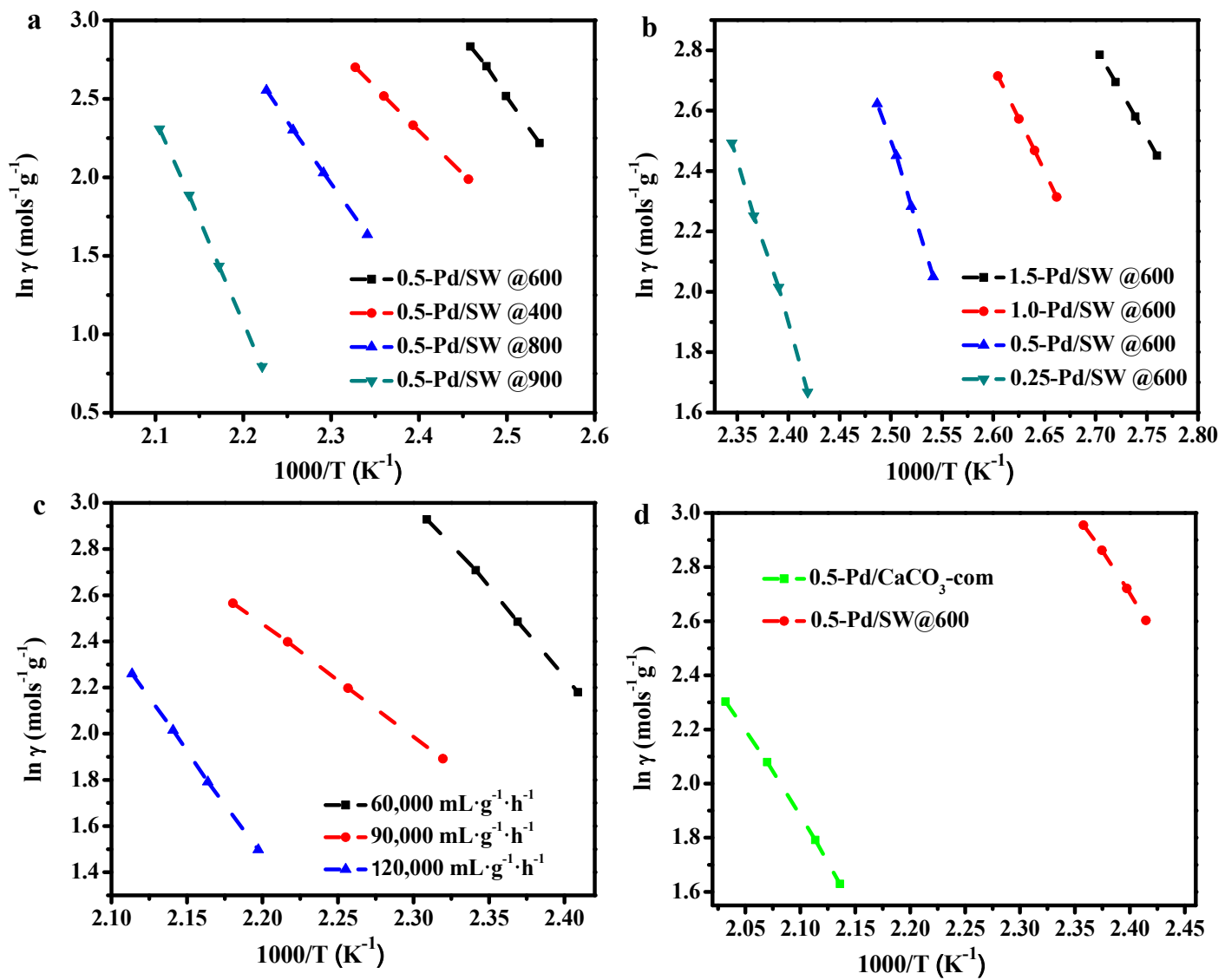

Figure S 5. Arrhenius graph of the tested catalysts 

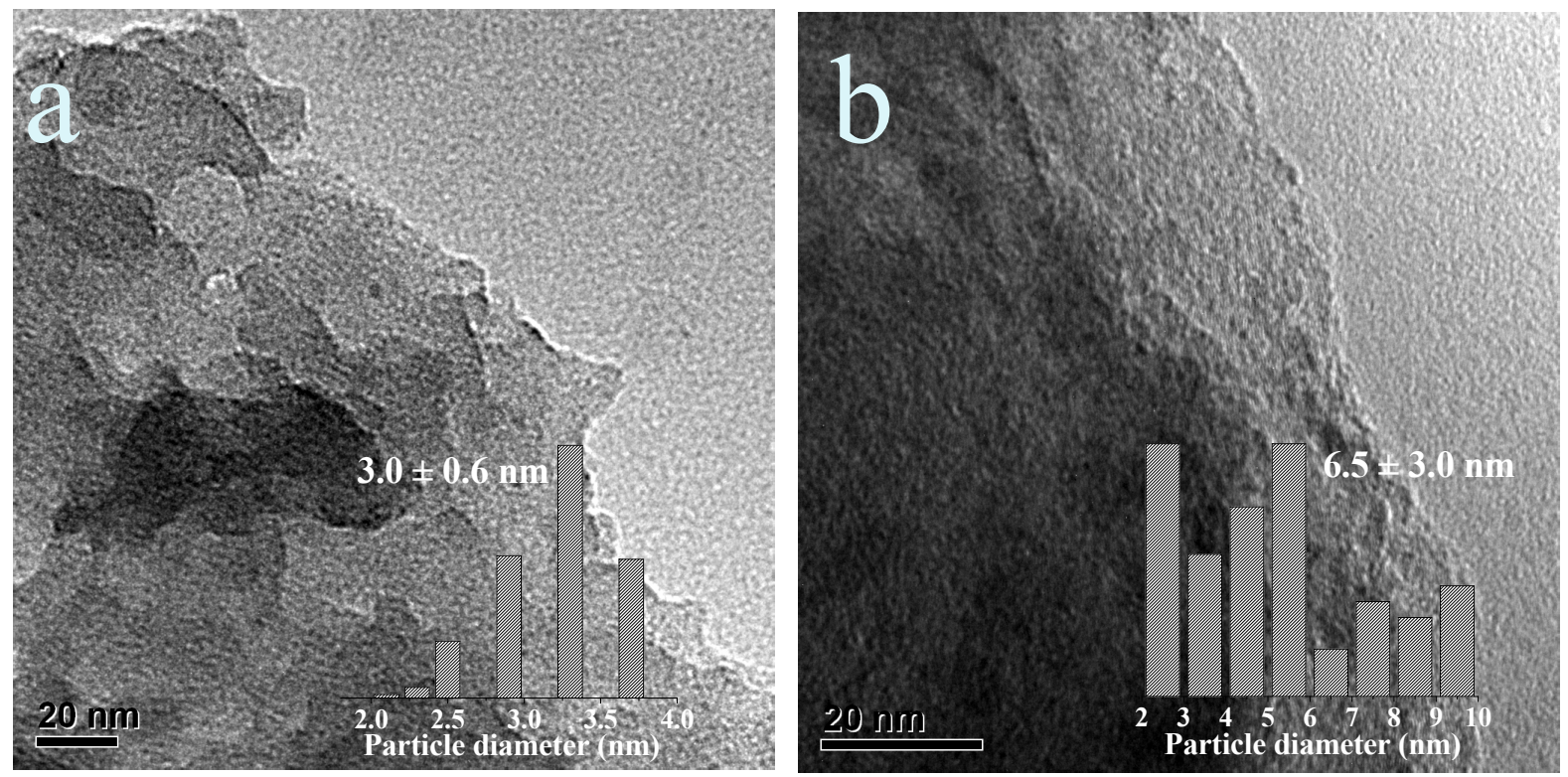

Figure S 6. TEM (a) 0.5-Pd/SW@600 and (b) 0.5-Pd/CaCO $3-\mathrm{Com}$ 

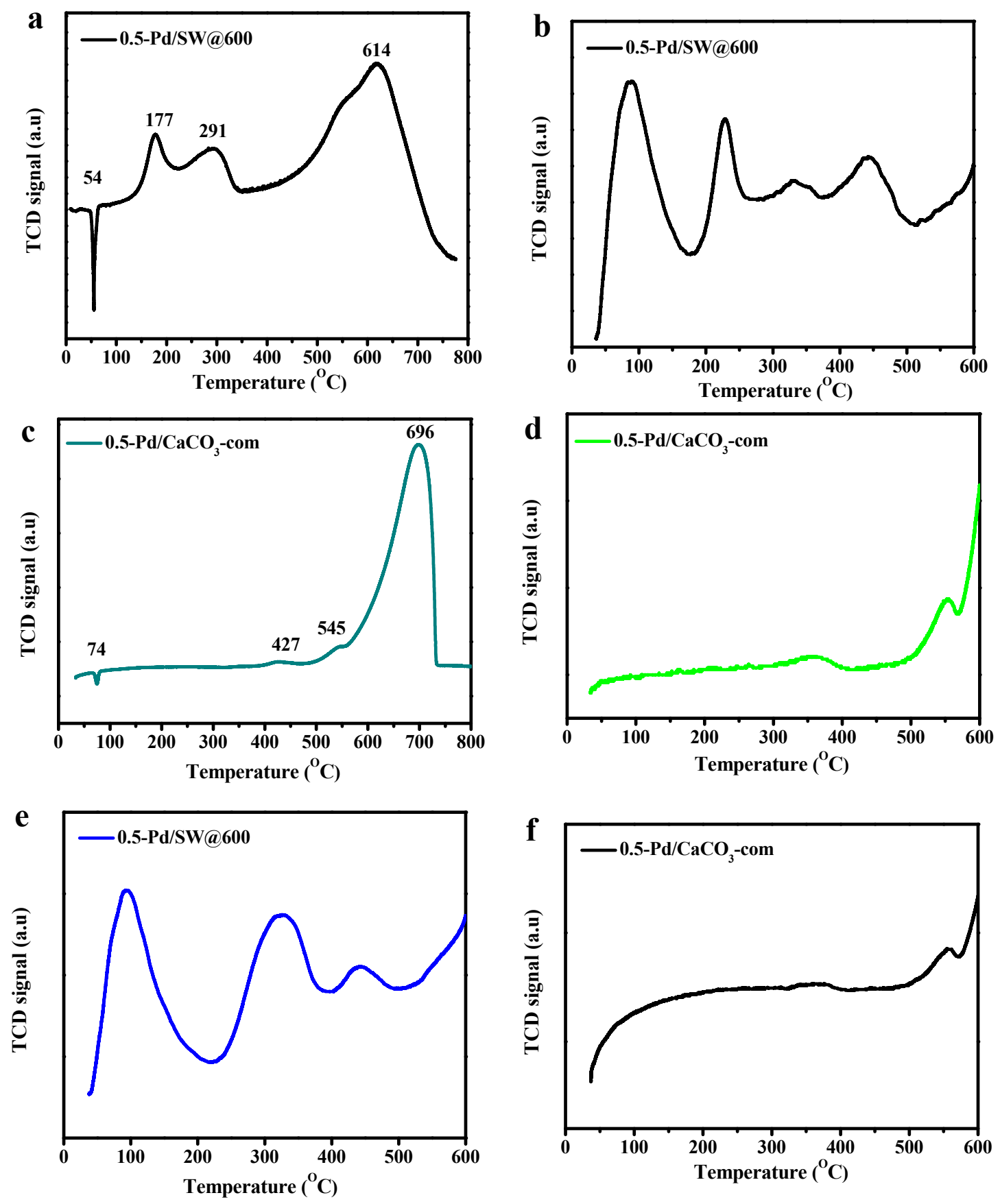

Figure S7. Comparison of the $\mathrm{H}_{2}$-TPR (a and c) $\mathrm{O}_{2}$-TPD ( $b$ and d) and $\mathrm{C}_{6} \mathrm{H}_{6}-\mathrm{TPD}$ of the 0.5-Pd/SW@600 and 0.5$\mathrm{Pd} / \mathrm{Com}$ 

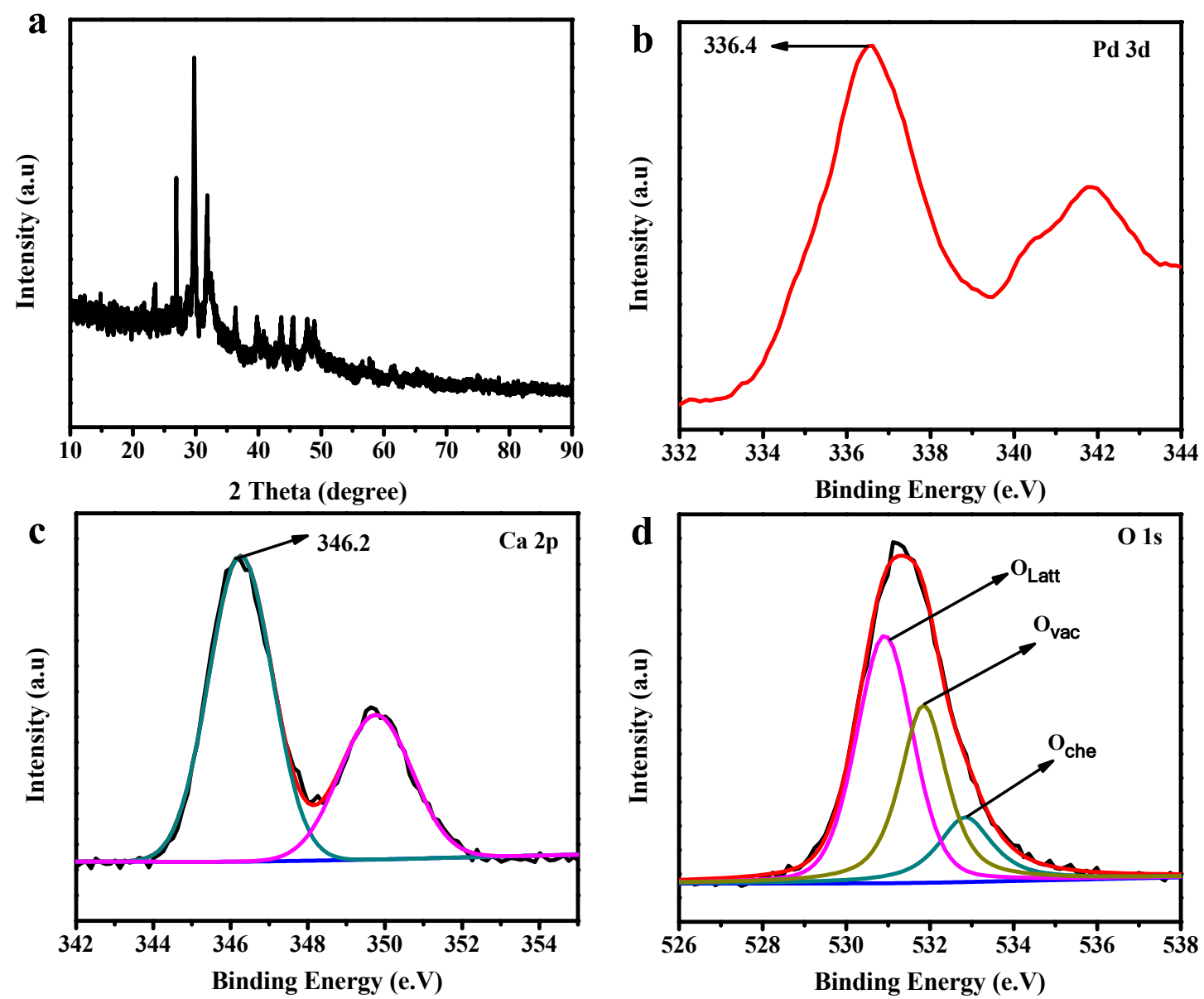

Figure S 8. XRD (a) and XPS analysis showing the (b) Pd 3d (c) Ca 2p and O1s region of the 0.5-Pd/SW@600 catalyst after $120 \mathrm{~h}$ of reaction at $240{ }^{\circ} \mathrm{C}$ and $\mathrm{GHSV}=60,000 \mathrm{~mL} \cdot \mathrm{g}^{-1} \cdot \mathrm{h}^{-1}$ 

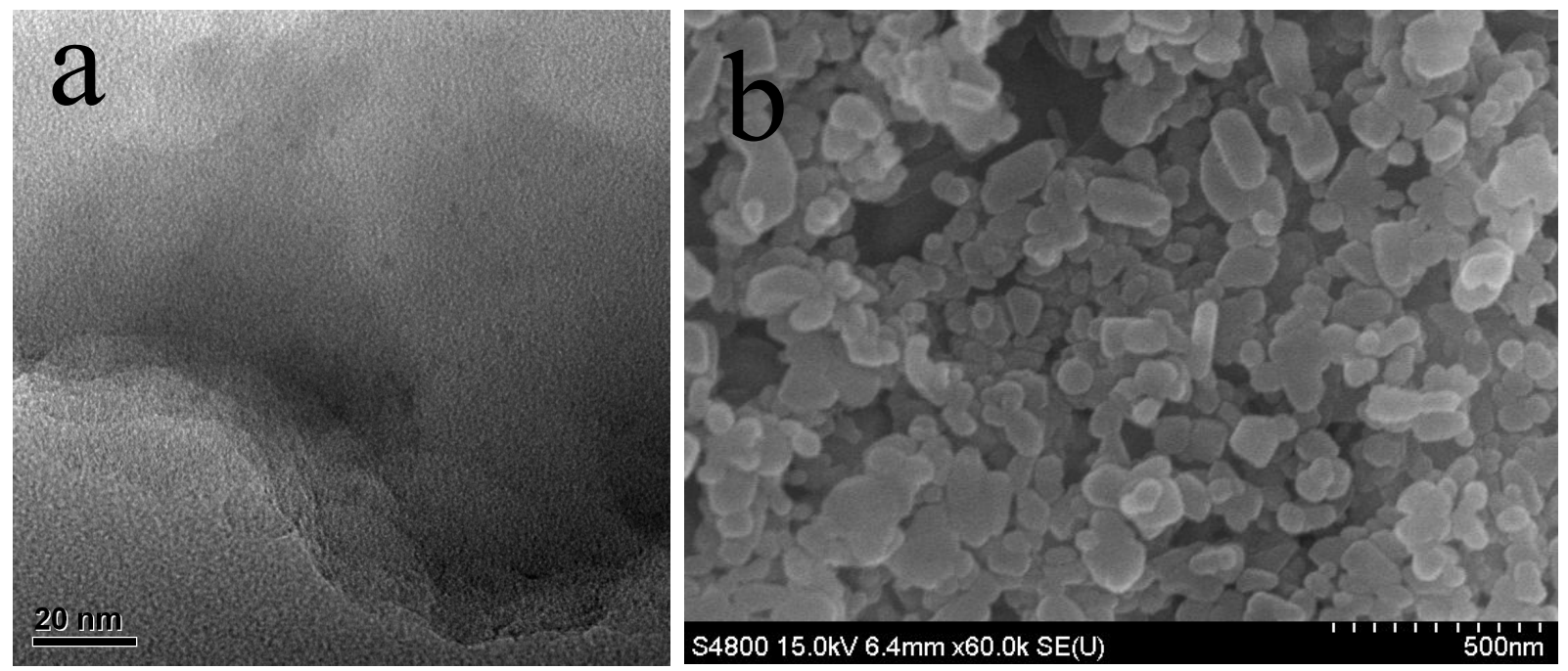

Figure S 9.TEM (a) and SEM analysis of the $0.5-\mathrm{Pd} / \mathrm{SW} @ 600$ catalyst after $120 \mathrm{~h}$ of reaction at $250{ }^{\circ} \mathrm{C}$ and GHSV= $60,000 \mathrm{~mL} \cdot \mathrm{g}^{-1} \cdot \mathrm{h}^{-1}$


Figure S 10. Effect of $\mathrm{CO}_{2}$ on conversion (a) and reuse-ability tests (b) of the 0.5-Pd/SW@600 catalyst ( $0.05 \mathrm{~g}$ of catalyst, $1000 \mathrm{ppm}$ of benzene, $20 \% \mathrm{O}_{2}$ and balance $\mathrm{N}_{2}, \mathrm{CO}_{2}$ on conversion was done at $\mathrm{GHSV}=60,000$ $\mathrm{mL} \cdot \mathrm{g}^{-1} \cdot \mathrm{h}^{-1}$ and the re-usability test GHSV $=20,000 \mathrm{~mL} \cdot \mathrm{g}^{-1} \cdot \mathrm{h}^{-1}$ ) N.B Figure $\mathrm{S} 10$ a was conducted in the presence of 10 and $20 \mathrm{vol} \%$ of high purity $\mathrm{CO}_{2}$. Before each reuse-ability test the temperature was allowed to cool down to $100{ }^{\circ} \mathrm{C}$ 
Table S1. Binding energies of the $\mathrm{Pd} 3 \mathrm{~d}, \mathrm{Ca} 2 \mathrm{p}$ and $\mathrm{O} 1 \mathrm{~s}$ region of different $\mathrm{Pd} / \mathrm{SW}$ catalysts

\begin{tabular}{|c|c|c|c|c|c|c|}
\hline Sample & $\operatorname{Pd~} 3 d_{5 / 2}$ & $\operatorname{Pd~3d~}_{3 / 2}$ & Ca $2 p_{3 / 2}$ & Ca $2 p_{1 / 2}$ & $\mathbf{O}_{\text {Latt }}$ & $\mathbf{O}_{\text {chem }}$ \\
\hline 0.5-Pd/SW@400 & 335.7 & 341.04 & 346.90 & 350.50 & 531.14 & 533.10 \\
\hline 0.5-Pd/SW@600 & 336.1 & 341.10 & 347.10 & 350.70 & 530.85 & 532.80 \\
\hline 0.5-Pd/SW@800 & 335.6 & 340.59 & 347.15 & 350.80 & 531.32 & 532.60 \\
\hline 0.5-Pd/SW@900 & 335.5 & 340.77 & 347.08 & 350.59 & 531.06 & 532.09 \\
\hline 1.5-Pd/SW@600 & 336.5 & 341.80 & 337.07 & 350.20 & 529.35 & 532.40 \\
\hline $1.0-P d / S W @ 600$ & 336.2 & 341.40 & 346.33 & 349.80 & 529.75 & 532.80 \\
\hline 0.25-Pd/SW@600 & 335.7 & 341.40 & 346.84 & 350.37 & 530.15 & 532.80 \\
\hline
\end{tabular}

Table S 2. Effect of $\mathrm{H}_{2} \mathrm{O}$ vapor on benzene conversion

\begin{tabular}{|c|c|}
\hline \multicolumn{2}{|c|}{15 vol $\% \mathrm{H}_{2} \mathrm{O}$ vapor on } \\
\hline Time (min) & Benzene conversion \\
\hline $\mathbf{0}$ & 100 \\
\hline 20 & 100 \\
\hline 40 & 100 \\
\hline 60 & 100 \\
\hline 80 & 100 \\
\hline $100-320$ & $95-80$ \\
\hline \multicolumn{2}{|c|}{15 vol $\% \mathrm{H}_{2} \mathrm{O}$ vapor off } \\
\hline 340 & 95 \\
\hline 360 & 95 \\
\hline 380 & 95 \\
\hline 400 & 95 \\
\hline 420 & 95 \\
\hline $440-480$ & 95 \\
\hline \multicolumn{2}{|c|}{25 vol $\% \% \mathrm{H}_{2} \mathrm{O}$ vapor on } \\
\hline $500-$ & $95-75$ \\
\hline 520 & 75 \\
\hline 540 & 75 \\
\hline 560 & 75 \\
\hline 580 & 75 \\
\hline $600-700$ & 75 \\
\hline \multicolumn{2}{|c|}{25 vol $\% \% \mathrm{H}_{2} \mathrm{O}$ vapor off } \\
\hline $720-800$ & 95 \\
\hline
\end{tabular}


Table $\mathbf{S}$ 3.Effect of High purity $\mathrm{CO}_{2}$ on conversion

\begin{tabular}{lc}
\hline & $\mathbf{1 0}$ vol \% High purity $\mathrm{CO}_{\mathbf{2}}$ on \\
\hline Time (min) & Benzene conversion \\
$\mathbf{0}$ & 100 \\
$\mathbf{2 0}$ & 100 \\
$\mathbf{4 0}$ & 100 \\
$\mathbf{6 0}$ & 100 \\
$\mathbf{8 0}$ & 100 \\
$\mathbf{1 0 0 - 3 2 0}$ & 96.6 \\
& $\mathbf{1 0}$ vol \% High purity $\mathrm{CO}_{\mathbf{2}}$ off \\
$\mathbf{3 4 0}$ & 99 \\
$\mathbf{3 6 0}$ & 99 \\
$\mathbf{3 8 0}$ & 99 \\
$\mathbf{4 0 0}$ & 99 \\
$\mathbf{4 2 0}$ & 99 \\
$\mathbf{4 4 0 - 4 8 0}$ & 99 \\
& $99-93$ \\
$\mathbf{5 0 0}$ & 93 \\
$\mathbf{5 2 0}$ & 93 \\
$\mathbf{5 4 0}$ & 93 \\
$\mathbf{5 6 0}$ & 93 \\
$\mathbf{5 8 0}$ & 93 \\
$\mathbf{6 0 0 - 7 0 0}$ & $\mathbf{2 0}$ vol \% High purity $\mathrm{CO}_{\mathbf{2}}$ on \\
& 98 \\
$\mathbf{7 2 0 - 8 0 0}$ & $\mathbf{2 0}$ vol \% High purity $\mathrm{CO}_{2}$ off \\
\hline
\end{tabular}


Table S 4. Recycling test over the 0.5-Pd/SW@600 catalyst

\begin{tabular}{|c|c|c|c|c|}
\hline Temperature & Cycle 1 & Cycle 2 & Cycle 3 & Cycle 4 \\
\hline 120 & 0 & 0 & 0 & 0 \\
\hline 140 & 7 & 6 & 5 & 5 \\
\hline 160 & 12 & 10 & 10 & 9 \\
\hline 180 & 25 & 23 & 22 & 22 \\
\hline 200 & 76 & 75 & 74 & 73 \\
\hline 230 & 92 & 92 & 91.8 & 92.5 \\
\hline 240 & 100 & 100 & 99.5 & 99.2 \\
\hline
\end{tabular}

Table S 5. Comparison of the $0.5-\mathrm{Pd} / \mathrm{SW} @ 600$ with others in literature

\begin{tabular}{|c|c|c|c|c|c|}
\hline Catalyst & Preparation method & $\begin{array}{c}\text { Concentration } \\
(\mathrm{ppm})\end{array}$ & Space velocity & $\mathbf{T}_{\mathbf{9 0 \%}}{ }^{\circ} \mathrm{C}$ & Ref. \\
\hline $\mathrm{Pt} / \mathrm{CeO}_{2}-1.8$ & glycol reduction & 1000 & $48,000 \mathrm{~mL} \cdot \mathrm{g}^{-1} \cdot \mathrm{h}^{-1}$ & 180 & 1 \\
\hline $\begin{array}{l}1-\mathrm{Pd} / \gamma-\mathrm{Al}_{2} \mathrm{O}_{3} \\
1-\mathrm{Pd} / \gamma-\mathrm{Al}_{2} \mathrm{O}_{3}\end{array}$ & $\begin{array}{l}\text { incipient wetness impregnation } \\
\text { incipient wetness impregnation } \\
\text { and hydrogen treatment }\end{array}$ & 1000 & $\begin{array}{l}15,000 \mathrm{~mL} \cdot \mathrm{g}^{-1} \cdot \mathrm{h}^{-1} \\
15,000 \mathrm{~mL} \cdot \mathrm{g}^{-1} \cdot \mathrm{h}^{-1}\end{array}$ & $\begin{array}{l}270 \\
240\end{array}$ & $\begin{array}{l}2 \\
2\end{array}$ \\
\hline 0.2Pd-Ni/SBA-15 & Co-impregnation & 1000 & $120,000 \mathrm{~mL} \cdot \mathrm{g}^{-1} \cdot \mathrm{h}^{-1}$ & 258 & 3 \\
\hline $\mathrm{Mn}_{2} \mathrm{Ni}_{1}$ mixed oxide & Nanocasting & 1000 & $120,000 \mathrm{~mL} \cdot \mathrm{g}^{-1} \cdot \mathrm{h}^{-1}$ & 249 & 4 \\
\hline $\mathbf{P t}-\mathrm{Pd} / \gamma-\mathrm{Al}_{2} \mathrm{O}_{3}$ & wetness impregnation & 1000 & $15,000 \mathrm{~h}^{-1}$ & 225 & 5 \\
\hline Pd/Ce/Al-PILC & impregnation & $<160$ & $20,000 \mathrm{~mL} \cdot \mathrm{g}^{-1} \cdot \mathrm{h}^{-1}$ & 250 & 6 \\
\hline 0.5-Pd/SW@600 & $\begin{array}{l}\text { Ethanol reduction and sol- } \\
\text { immobilization }\end{array}$ & 1000 & $120,000 \mathrm{~mL} \cdot \mathrm{g}^{-1} \cdot \mathrm{h}^{-1}$ & 260 & $\begin{array}{l}\text { This } \\
\text { work }\end{array}$ \\
\hline 0.5-Pd/SW@600 & $\begin{array}{l}\text { Ethanol reduction and sol- } \\
\text { immobilization }\end{array}$ & 1000 & $20,000 \mathrm{~mL} \cdot \mathrm{g}^{-1} \cdot \mathrm{h}^{-1}$ & 220 & This work \\
\hline
\end{tabular}


References

1. $\quad$ Peng, R.; Li, S.; Sun, X.; Ren, Q.; Chen, L.; Fu, M.; Wu, J.; Ye, D., Size effect of Pt nanoparticles on the catalytic oxidation of toluene over Pt/CeO2 catalysts. Applied Catalysis B: Environmental2018, 220, 462-470.

2. Kim, S. C.; Shim, W. G., Properties and performance of Pd based catalysts for catalytic oxidation of volatile organic compounds. Applied Catalysis B: Environmental 2009, 92, 429436.

3. Tang, W.; Deng, Y.; Chen, Y., Promoting effect of acid treatment on Pd-Ni/SBA-15 catalyst for complete oxidation of gaseous benzene. Catalysis Communications 2017, 89, 86-90.

4. Tang, W.; Li, J.; Wu, X.; Chen, Y., Limited nanospace for growth of Ni-Mn composite oxide nanocrystals with enhanced catalytic activity for deep oxidation of benzene. Catalysis Today 2015, 258, 148-155.

5. Kim, H. S.; Kim, T. W.; Koh, H. L.; Lee, S. H.; Min, B. R., Complete benzene oxidation over Pt-Pd bimetal catalyst supported on $\gamma$-alumina: influence of Pt-Pd ratio on the catalytic activity. Applied Catalysis A: General 2005, 280, 125-131.

6. Zuo, S.; Zhou, R., Influence of synthesis condition on pore structure of Al pillared clays and supported Pd catalysts for deep oxidation of benzene. Microporous and Mesoporous Materials 2008, 113, 472-480. 